\title{
EFEITO DA CONCENTRAÇÃO DE GLICEROL SOBRE A PRODUÇÃO DE 2,3-BUTANODIOL POR Enterobacter aerogenes
}

\author{
V. GIRARDI ${ }^{1}$, B. BARSÉ ${ }^{1}$, C. M. BECKER ${ }^{1}$, L. MENEGHEL ${ }^{1}$, A. P. TORRES ${ }^{2}$, M. P. \\ SOUSA $^{2}$, L. L. BEAL ${ }^{3}$, E. MALVESSI ${ }^{1}$, M. M. da SILVEIRA ${ }^{1}$ \\ 1 Universidade de Caxias do Sul, Instituto de Biotecnologia, Caxias do Sul-RS \\ 2 Petrobrás, Centro de Pesquisa e Desenvolvimento (CENPES), Rio de Janeiro- RJ \\ 3 Universidade Caxias do Sul, Laboratório de Tecnologias Ambientais, Caxias do Sul-RS \\ E-mail para contato: vgirard1@ucs.br
}

\begin{abstract}
RESUMO - 2,3-Butanodiol pode ser obtido via processo fermentativo utilizando glicerol como substrato. O objetivo deste trabalho foi avaliar o efeito da concentração de glicerol sobre o crescimento e a produção de butanodiol por Enterobacter aerogenes. Foram avaliadas concentrações iniciais de glicerol $\left(\mathrm{S}_{0}\right)$ de 20, 40, 60, 80 e $100 \mathrm{~g} / \mathrm{L}$. Os valores de conversão de substrato em células ( $\mathrm{Y}_{\mathrm{X} / \mathrm{S}}$ ) obtidos foram $0,13,0,05,0,03,0,03$ e $0,01 \mathrm{~g} / \mathrm{g}$. Em termos de conversão de substrato em produto $\left(\mathrm{Y}_{\mathrm{P} / \mathrm{S}}\right)$, os valores calculados foram $0,26,0,31,0,33,0,42 \mathrm{e}$ $0,45 \mathrm{~g} / \mathrm{g}$, respectivamente, com maior rendimento em relação ao máximo teórico (93\%) para $\mathrm{S}_{0}$ de $100 \mathrm{~g} / \mathrm{L}$. Em razão da limitação de oxigênio dissolvido, especialmente em cultivos de maior duração, houve um decréscimo do rendimento em ATP e consequentemente, menor formação de biomassa; por outro lado a síntese de produto aumentou devido à necessidade de reoxidação de NADH pela via fermentativa.
\end{abstract}

\section{INTRODUÇÃO}

2,3-Butanodiol pode ser utilizado na indústria de alimentos, de cosméticos, produtos farmacêuticos e plásticos (Syu, 2001; Celinska \& Grajek, 2009). Além disso, o elevado calor de combustão $(\approx 27 \mathrm{KJ} / \mathrm{g})$ se compara favoravelmente ao do etanol $(29 \mathrm{KJ} / \mathrm{g})$, indicando a possibilidade de sua utilização como combustível (Flickinger, 1980).

Entre os microrganismos produtores de 2,3-butanodiol, destacam-se bactérias anaeróbias facultativas dos gêneros Klebsiella e Enterobacter (Zeng \& Sabra, 2011; Jung et. $a l, 2013$, Yen et. al, 2014). No processo de produção podem ser empregadas diversas fontes de carbono, como glicose, xilose, lactose e sacarose. Além destes substratos, o glicerol, resíduo da produção de biodiesel, também é metabolizado por estes microrganismos. Com o crescimento da produção de biodiesel, aumenta a preocupação com o destino do glicerol excedente, já que é formado na base de $10 \%$ em relação à produção do biocombustível (Silva et al., 2009; Quispe et al. 2013). Para evitar futuros problemas derivados da acumulação do 
glicerol, torna-se necessária a busca de alternativas para o uso deste resíduo industrial (Ooi et $a l .$, 2004).

Com vistas a um futuro processo fermentativo de produção de 2,3-butanodiol a partir de glicerol, a obtenção de alta concentração de produto no meio é de extrema importância, especialmente considerando a etapa de recuperação. Para isto, entretanto, é necessário que elevadas concentrações de glicerol sejam utilizadas no processo, o que pode acarretar em perda de produtividade no processo em razão de uma possível inibição pelo substrato. (Silveira et. al, 1998; Garcia, 2006)

Neste contexto, o objetivo deste trabalho foi avaliar o efeito da concentração de glicerol, entre 20 e 100g/L, sobre o crescimento de Enterobacter aerogenes e a produção de 2,3-butanodiol.

\section{MATERIAIS E MÉTODOS}

\subsection{Microrganismo}

Foi utilizada a bactéria E. aerogenes ATCC 13048, adquirida do Centro de Culturas Tropicais André Tosello (São Paulo-SP, Brasil), mantida em ágar nutriente, a $4^{\circ} \mathrm{C}$. Os inóculos foram preparados em frascos de $500 \mathrm{~mL}$, cobertos com manta de algodão e gaze, contendo $100 \mathrm{~mL}$ de meio com $20 \mathrm{~g} / \mathrm{L}$ de glicerol em $\mathrm{pH}$ inicial 6,5. Os frascos foram mantidos sob agitação recíproca, a $300 \mathrm{rpm}$ por 10 horas, a $37^{\circ} \mathrm{C}$. Posteriormente, no biorreator, o meio de cultivo previamente esterilizado foi inoculado com o volume de necessário relativo à concentração celular inicial de cerca $0,2 \mathrm{~g} / \mathrm{L}$.

\subsection{Meio de cultivo}

O meio líquido utilizado nos inóculos e nos ensaios em biorreator foi baseado no descrito por Pirt e Callow (1958), com a seguinte composição, em (g/L): glicerol comercial, $20,40,60,80$ e $100 ; \mathrm{MgSO}_{4} \cdot 7 \mathrm{H}_{2} \mathrm{O}, 0,3 ; \mathrm{CaCl}_{2} \cdot 6 \mathrm{H}_{2} \mathrm{O}, 0,09 ; \mathrm{FeSO}_{4} \cdot 7 \mathrm{H}_{2} \mathrm{O}, 0,023$; $\mathrm{ZnSO}_{4} .4 \mathrm{H}_{2} \mathrm{O}, 0,0038 ;\left(\mathrm{NH}_{4}\right)_{2} \mathrm{SO}_{4}, 7,2 ;\left(\mathrm{NH}_{4}\right)_{2} \mathrm{HPO}_{4}, 6,0$. A solução de sais nutrientes e de glicerol foram autoclavados separadamente.

\subsection{Condições experimentais}

Foram realizados ensaios em biorreator de bancada New Brunswick (EUA) com 4,0L de meio. Os cultivos foram conduzidos em batelada, a $37^{\circ} \mathrm{C}$ e controle automático de $\mathrm{pH}$ em 5,5 pela adição de $\mathrm{NaOH} 7 \mathrm{~mol} / \mathrm{L}$. Em todos os cultivos, o fluxo de ar empregado foi de 2,0L/min e a frequência dos agitadores de $500 \mathrm{rpm}$, sendo estes parâmetros mantidos até o final do processo. Amostras foram coletadas para a quantificação de biomassa, do consumo de substrato e da formação de produtos.

\subsection{Métodos Analíticos}

A concentração de biomassa foi determinada diretamente por gravimetria ou por leitura da absorbância de suspensões celulares, a 520nm, convertida em massa/volume a partir de 
uma curva de calibração. O consumo de glicerol foi avaliado pelo método descrito por Carra (2012), adaptado para glicerol.

Os principais produtos da fermentação, 2,3-butanodiol e acetoína foram determinados por cromatografia líquida de alto desempenho (CLAD), em cromatógrafo Agilent

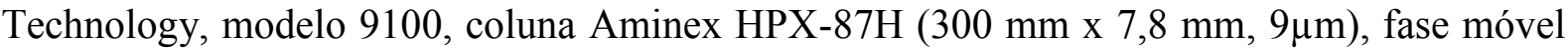
$\mathrm{H}_{2} \mathrm{SO}_{4} 0,0005 \mathrm{M}$, vazão de $0,6 \mathrm{~mL} / \mathrm{min}$, volume de injeção de $50 \mu 1$, a $60^{\circ} \mathrm{C}$. Devido ao equilíbrio observado na formação entre 2,3-butanodiol e acetoína, a análise destes resultados foi realizada em conjunto (Jansen et al., 1984; Silveira et al., 1998).

\subsection{Métodos usados em cálculos}

Em meio isento de células o coeficiente volumétrico de transferência de oxigênio $\left(\mathrm{K}_{\mathrm{L}} \mathrm{a}\right)$ foi determinado utilizando-se o método estático definido por Moo-Young \& Blanch (1989) em que um eletrodo de oxigênio dissolvido é acoplado ao reator para registrar os valores de porcentagem de oxigênio na saturação. $\mathrm{O} \mathrm{K}_{\mathrm{L}}$ a foi calculado a partir da equação:

$$
K_{L} a=-\frac{1}{t} \cdot \ln \frac{1-\beta}{1-\alpha}
$$

onde $\alpha$ e $\beta$ são as frações da saturação em oxigênio para as condições inicial e final avaliadas.

As máximas velocidades específicas de crescimento $\left(\mu_{\mathrm{xmax}}\right)$ foram determinadas, a partir das concentrações celulares $(\mathrm{X})$ medidas durante a fase exponencial de crescimento, correspondendo ao coeficiente angular de uma reta $\ln \mathrm{X}=\mathrm{f}$ (tempo) para esta fase.

Os fatores de conversão de substrato em células $\left(\mathrm{Y}_{\mathrm{X} / \mathrm{S}}\right)$ e em produto $\left(\mathrm{Y}_{\mathrm{P} / \mathrm{S}}\right)$ foram calculados a partir dos valores iniciais e finais de concentração celular, substrato e produto.

\section{RESULTADOS}

Neste trabalho foram testadas concentrações iniciais de glicerol $\left(\mathrm{S}_{0}\right)$ de 20, 40, 60, $80 \mathrm{e}$ $100 \mathrm{~g} / \mathrm{L}$ sobre o crescimento de E. aerogenes e a produção de 2,3-butanodiol. Na Figura 1, são mostrados os perfis cinéticos dos cultivos com $S_{0}$ de 20 a $100 \mathrm{~g} / \mathrm{L}$. Nos testes com $S_{0}$ de 20 e 40 g/L (Figura 1A e 1B), concentrações finais de biomassa $\left(X_{f}\right)$ de 2,7 e 2,1 g/L foram determinados em 8 e 24h, respectivamente, com total consumo de glicerol. Nestas condições, foi observado um período aproximado de 5 e 7 horas de duração da fase exponencial de crescimento, respectivamente, seguidos de fase estacionária até o final do ensaio. Na Figura $1 \mathrm{C}, 1 \mathrm{D}$ e 1E, são mostrados os perfis cinéticos dos cultivos com $\mathrm{S}_{0}$ de 60 a $100 \mathrm{~g} / \mathrm{L}$. Valores de $X_{\mathrm{f}}$ de 2,2, 2,0 e 1,7 g/L foram medidos, com praticamente total consumo de glicerol, com exceção do cultivo com $\mathrm{S}_{0}$ de $60 \mathrm{~g} / \mathrm{L}$, em que mediu-se, no tempo final do ensaio, 3,6 g/L de glicerol (Tabela 1). Na média, nestes ensaios, a fase estacionária de crescimento celular foi observada a partir de $7 \mathrm{~h}$ de cultivo.

Devido ao intenso metabolismo celular, foi constatada queda acentuada do percentual de oxigênio na saturação em todos os cultivos (Figura 1). No cultivo com $S_{0}$ de $20 \mathrm{~g} / \mathrm{L}$, 
percentual de oxigênio nulo foi observado após cerca de 2 horas de cultivo e em torno de 3 horas nas demais condições (Figura 1 e Tabela 1).
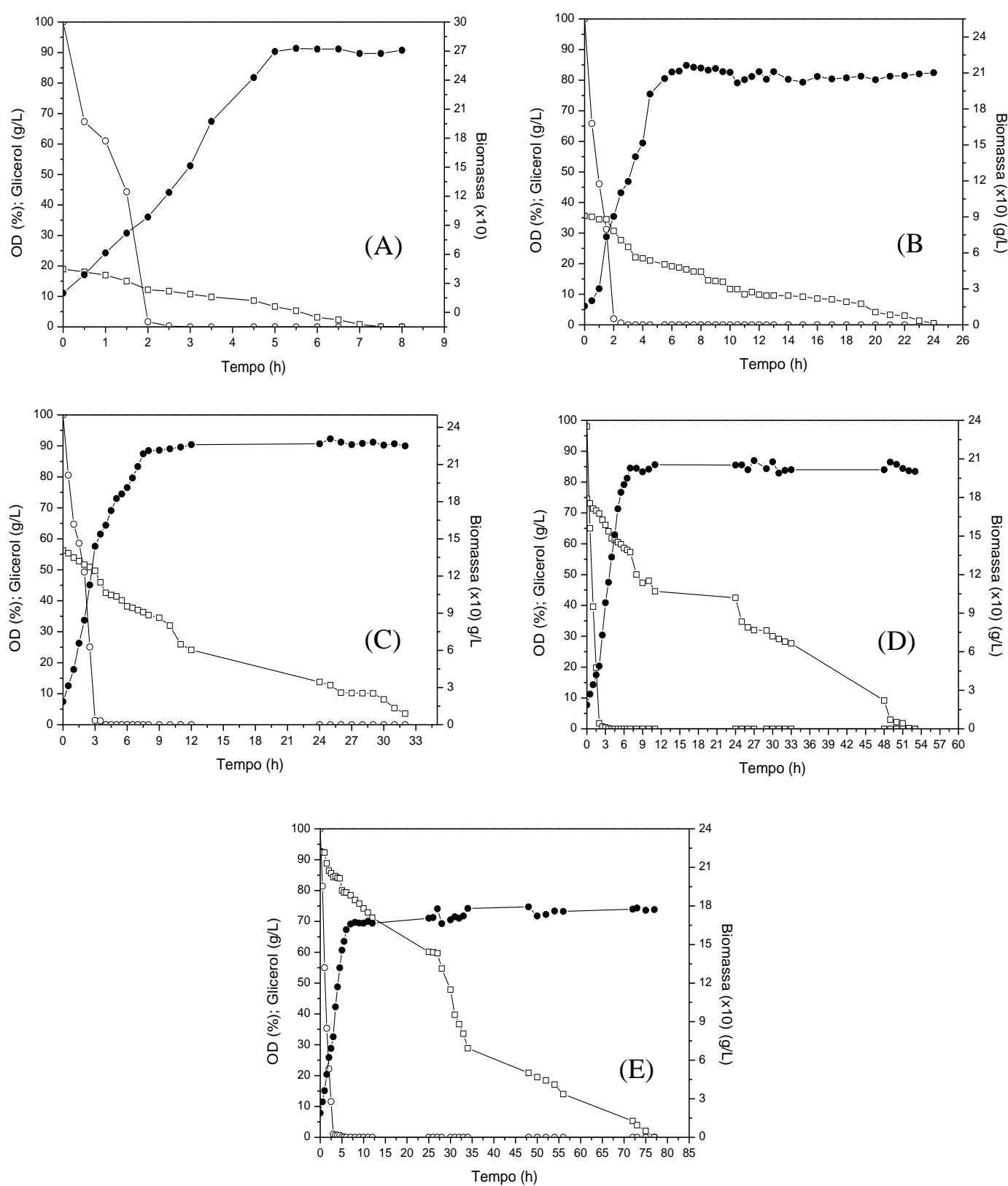

Figura 1: Variação das concentrações de biomassa, glicerol e oxigênio dissolvido (OD), com o tempo de cultivo de Enterobacter aerogenes em biorreator de bancada. (A) $20 \mathrm{~g} / \mathrm{L}$; (B) $40 \mathrm{~g} / \mathrm{L}$; (C) $60 \mathrm{~g} / \mathrm{L}$; (D) $80 \mathrm{~g} / \mathrm{L}$; (E) $100 \mathrm{~g} / \mathrm{L}$ de glicerol. (•) Biomassa; ( $\square$ ) glicerol; (०) $\mathrm{O}_{2}$ dissolvido 
Foram realizadas medidas de $\mathrm{K}_{\mathrm{L}} \mathrm{a}$, em meio isento de células nas condições dos ensaios realizados (500 rpm e fluxo de ar $2 \mathrm{~L} / \mathrm{min}$ ). Os valores estimados foram 70, 66, 63, $61,61 \mathrm{~h}^{-1}$, respectivamente para $\mathrm{S}_{0} 20,40,60,80100 \mathrm{~g} / \mathrm{L}$. Observou-se decréscimo dos valores de $\mathrm{K}_{\mathrm{L}} \mathrm{a}$, com aumento de $\mathrm{S}_{0}$, possivelmente devido ao aumento da viscosidade do meio nas concentrações superiores de glicerol.

Os valores de fator de conversão de substrato em biomassa $\left(\mathrm{Y}_{\mathrm{X} / \mathrm{S}}\right)$ e máxima velocidade de crescimento $\left(\mu_{\mathrm{xmax}}\right)$ são apresentados na Tabela 1. Valores decrescentes de $\mathrm{Y}_{\mathrm{X} / \mathrm{S}}$ foram calculados com o aumento de $\mathrm{S}_{0}$. Isto se deve ao fato de o metabolismo respiratório bacteriano ter sido progressivamente reduzido nos cultivos de maior duração, em razão da limitação de oxigênio dissolvido, provocando um decréscimo do rendimento em ATP e, consequentemente, menor crescimento microbiano. Com relação a $\mu_{\mathrm{xmax}}$, foi observado um decréscimo nos valores com o aumento de $S_{0}$, mais evidente com $S_{0}$ de $100 \mathrm{~g} / \mathrm{L}$, indicando efeito de inibição do crescimento pelo substrato.

Tabela 1: Resultados gerais dos cultivos de Enterobacter aerogenes em biorreator de bancada sob diferentes concentrações de glicerol, em termos de crescimento celular.

\begin{tabular}{cccccccc}
\hline $\begin{array}{c}\text { Glicerol } \\
(\mathrm{g} / \mathrm{L})\end{array}$ & $\begin{array}{c}\mathrm{X}_{\mathrm{f}} \\
(\mathrm{g} / \mathrm{L})\end{array}$ & $\begin{array}{c}\mathrm{S}_{0} \\
(\mathrm{~g} / \mathrm{L})\end{array}$ & $\begin{array}{c}\mathrm{S}_{\mathrm{f}} \\
(\mathrm{g} / \mathrm{L})\end{array}$ & $\begin{array}{c}\mathrm{t}_{\mathrm{f}} \\
(\mathrm{h})\end{array}$ & $\begin{array}{c}\mathrm{Y}_{\mathrm{X} / \mathrm{S}} \\
(\mathrm{g} / \mathrm{g})\end{array}$ & $\begin{array}{c}\mu_{\mathrm{xmax}} \\
\left(\mathrm{h}^{-1}\right)\end{array}$ & $\begin{array}{c}\text { Tempo de O2 } \\
\text { dissolvido }(\mathrm{h})\end{array}$ \\
\hline 20 & 2,7 & 18,9 & 0 & 8 & 0,13 & 0,63 & 2 \\
40 & 2,1 & 35,6 & 0,51 & 24 & 0,05 & 0,61 & 2,5 \\
60 & 2,3 & 56,2 & 3,57 & 32 & 0,03 & 0,59 & 3 \\
80 & 2,0 & 74,6 & 0 & 53 & 0,03 & 0,58 & 3,5 \\
100 & 1,8 & 92,7 & 0 & 77 & 0,01 & 0,49 & 3,5 \\
\hline
\end{tabular}

$\mathrm{X}_{\mathrm{f}}$ - concentração celular final; $\mathrm{S}_{0}$ - concentração inicial de glicerol; $\mathrm{S}_{\mathrm{f}}$ - concentração final

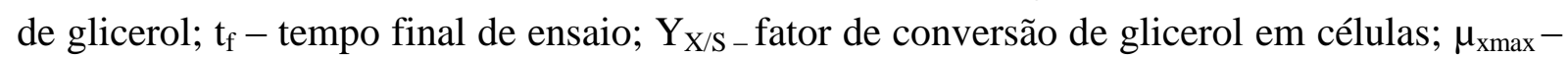
máxima velocidade específica de crescimento celular.

Na Tabela 2, são apresentados os resultados gerais em relação à produção de 2,3butanodiol. Como pode ser observado, houve aumento na concentração de produto, no fator de conversão em butanodiol ( $\left.\mathrm{Y}_{\mathrm{P} / \mathrm{S}}\right)$ e na produtividade volumétrica $(\mathrm{p})$ com o incremento em $\mathrm{S}_{0}$. Valor superior de rendimento em relação ao máximo teórico, foi obtido no ensaio com $\mathrm{S}_{0}$ de $100 \mathrm{~g} / \mathrm{L}$, de 93\%. Em estudos realizados por Silveira et. al (1998), em que foi usada a bactéria $K$. pneumoniae, e sacarose $(95 \mathrm{~g} / \mathrm{L})$ como fonte carbono, rendimento semelhante foi obtido. 
Tabela 2: Resultados gerais dos cultivos de Enterobacter aerogenes em biorreator de bancada sob diferentes concentrações de glicerol, em termos de formação de 2,3-butanodiol.

\begin{tabular}{ccccc}
\hline $\begin{array}{c}\text { Glicerol } \\
(\mathrm{g} / \mathrm{L})\end{array}$ & $\begin{array}{c}\text { Butanodiol } \\
(\mathrm{g} / \mathrm{L})\end{array}$ & $\begin{array}{c}\mathrm{Y}_{\mathrm{P} / \mathrm{S}} \\
(\mathrm{g} / \mathrm{g})\end{array}$ & $\begin{array}{c}\rho \\
(\%)\end{array}$ & $\begin{array}{c}\mathrm{p} \\
(\mathrm{g} / \mathrm{L} / \mathrm{h})\end{array}$ \\
\hline 20 & 5,0 & 0,26 & 53 & 0,62 \\
40 & 11,0 & 0,31 & 64 & 0,46 \\
60 & 17,8 & 0,33 & 69 & 0,55 \\
80 & 31,6 & 0,42 & 86 & 0,59 \\
100 & 42,2 & 0,45 & 93 & 0,54 \\
\hline
\end{tabular}

$\mathrm{Y}_{\mathrm{P} / \mathrm{S}}$ - fator de conversão de glicerol em butanodiol; $\rho$ - rendimento em 2,3-butanodiol / acetoína em relação ao máximo teórico $(0.489$ g/g glicerol); $\mathrm{p}$ - produtividade volumétrica.

Apesar de decréscimo de $\mathrm{Y}_{\mathrm{X} / \mathrm{S}}$ com maiores $\mathrm{S}_{0}$, a formação de produto é favorecida nestes casos devido à limitação de oxigênio, condição que predomina em cultivos com maior duração, ou seja, naqueles $S_{0}$ superiores. Nestas condições, há menor obtenção de energia na forma de ATP, com menor crescimento, levando então ao aumento do fluxo de carbono na direção da produção de 2,3-butanodiol. A formação deste produto está relacionada à necessidade de reoxidação de NADH produzidos na conversão de glicerol em piruvato para obtenção de energia metabólica para a manutenção celular (Amaral et al., 2009). Deste modo, uma estratégia para aumentar a concentração de glicerol no cultivo, sem prejudicar o crescimento microbiano, seria a condução dos ensaios com emprego de frequências de agitação e fluxos de ar variáveis durante o processo. Em um primeiro momento, o maior suprimento de oxigênio - maior frequência de agitação e/ou fluxo de ar - favoreceria a formação de biomassa e, posteriormente, com menor velocidade de dissolução de $\mathrm{O}_{2}$, a formação de 2,3-butanodiol seria priorizada. Ji et. al (2009), em estudos empregando Klebsiella oxytoca em meio contendo $200 \mathrm{~g} / \mathrm{L}$ de glicose, relatam o emprego de frequência dos agitadores de $300 \mathrm{rpm}$ nas primeiras 15 horas de cultivo e, após este período, redução para $200 \mathrm{rpm}$. Nestas condições, os autores relatam a obtenção de 95,5 g/L de butanodiol.

\section{CONCLUSÕES}

O estudo do efeito da concentração inicial de glicerol mostrou que este parâmetro influencia o crescimento de E. aerogenes e a produção de 2,3-butanodiol. $O$ emprego de $\mathrm{S}_{0}$ de $100 \mathrm{~g} / \mathrm{L}$ possibilitou a obtenção de resultados superiores em termos de formação de produto; no entanto, concentração inferior de biomassa foi obtida. Além disso, foi constatado necessidade de uma etapa de limitação de oxigênio para obtenção de maiores concentrações de 2,3-butanodiol. Os resultados indicam, ainda, a necessidade de estudos em regime descontínuo alimentado com o fim de evitar problemas de inibição do crescimento celular e possibilitar o aumento da concentração final de produto.

\section{AGRADECIMENTOS}

À Universidade de Caxias do Sul, PETROBRAS e FAPERGS pelo apoio na realização deste trabalho. 


\section{REFERÊNCIAS BIBLIOGRÁFICAS}

AMARAL, P. F. F.; FERREIRA, T. F.; FONTES, G. C.; COELHO, M. A. Z. Glycerol valorization: New biotechnological routes. Food and Bioproducts Processing, v.87 ; p.179-186, 2009.

CARRA, S. Estudo cinético da produção de ácido lactobiônico e sorbitol por enzimas periplasmáticas de Zymomonas mobilis. Dissertação de Mestrado. Universidade de Caxias do Sul, Programa de PósGraduação em Biotecnologia, Caxias do Sul, RS, 2012.

CELINSKA, E.; GRAJEK, W. Biotechnological production of 2,3-butanediol-Current state and prospects. Biotechnology Advances, v.27; p.715-725, 2009.

FLICKINGER, M. C. Current biological research in conversion of cellulosic carbohydrates into liquid fuels: how far have we come? Biotechnology and Bioengineering, v.22; p.27-48, 1980.

GARCIA, A. D. Produção fermentativa de 2,3-butanodiol a partir de hidrolisado hemicelulósico de eucalipto. Tese de Doutorado. Faculdade de Engenharia Química de Lorena. Programa de PósGraduação em Biotecnologia Industrial, Lorena, SP, 2006.

JANSEN, N. B.; FLICKINGER, M. C.; TSAO, G. T. Production of 2,3-butanediol from D-xylose by Klebsiella oxytoca ATCC 8724. Biotechnology and Bioengineering, v. 26; p.362-369, 1984.

JI, X.J.; HUANG, H.; OUYANG, P.K. Microbial 2,3-butanediol production: A state-of-the-art review. Biotechnology Advances. v.29; p. 351-364, 2011.

JI, X.J.; HUANG, H.; DU, J.; ZHU, J.G.; REN, L. J.; HU, N.; LI, S. Enhanced 2,3-butanediol production by Klebsiella oxytoca using a two-stage agitation speed control strategy. Bioresource Technology, v.100; p. 3410-3414, 2009.

JUNG, M.Y.; PARK, B.S.; LEE, J.; OH, M.K. Engineered Enterobacter aerogenes for efficient utilization of sugarcane molasses in 2,3-butanediol production. Bioresource Technology, v. 139; p. 21$27,2013$.

MOO-YOUNG, M. e BLANCH, H. W. Z Transport phenomena and bioreactor design. Basic Biotechnology. v. 3; p. 135-151, 1989.

OOI, T. L.; YONG, K. C.; HAZIMAH, A.H.; DZULKEFLY, K. Glycerol Residue - A Rich Source of Glycerol and Medium Chain Fatty Acids. Journal of Oleo Science. v.53; p. 29-33, 2004

PIRT, S. J.; CALLOW, D. S. Production of 2,3-butanediol by Aerobacter aerogenes in a single stage process. Journal of Applied Bacteriology, v. 21; p. 188-205, 1958

QUISPE, C. A. G.; CORONADO, C. J. R.; CARVALHO, J. A. Glycerol: Production, consumption, prices, characterization and new trends in combustion. Renewable and Sustainable Energy Reviews, v. 27; p. 75-93, 2013. 
SILVA, G.P.; MACK, M.; CONTIERO, J. Glycerol: A promising and abundant carbon source for industrial microbiology. Biotechnology Advances, v.27; p. 30-39, 2009.

SILVEIRA, M. M.; MOLINA, M. A. B.; PRATA, A.M.R.; SCHMIDELL, W. Production of 2,3butanediol from sucrose by Klebsiella pneumoniae NRRL B199 in batch and fed-batch reactors. Brazilian Archives of Biology and Technology, v. 41; p. 329-334,1998.

YEN, H.W.; LI, F.T.; CHANG, J.S. The influences of $\mathrm{pH}$ control strategies on the distribution of 1,3propanediols and 2,3-butanediols production by an isolated indigenous Klebsiella sp. Ana-WS5. Bioresource Technology, v.159; p. 292-296, 2014. 